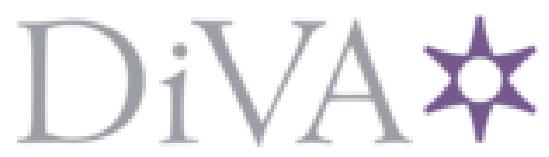

http://www.diva-portal.org

This is the published version of a paper presented at Conference on Nanotechnology VI, APR 24-25, 2013, Grenoble, France.

Citation for the original published paper:

Sangghaleh, F., Bruhn, B., Sychugov, I., Linnros, J. (2013)

Optical absorption cross section and quantum efficiency of a single silicon quantum dot.

In: Nanotechnology VI (pp. 876607-). SPIE - International Society for Optical Engineering

Proceedings of SPIE

http://dx.doi.org/10.1117/12.2017483

N.B. When citing this work, cite the original published paper.

Permanent link to this version:

http://urn.kb.se/resolve?urn=urn:nbn:se:kth:diva-128505 


\title{
Optical absorption cross section and quantum efficiency of a single silicon quantum dot
}

\author{
F. Sangghaleh*a , B. Bruhn ${ }^{\mathrm{a}}$, I. Sychugov ${ }^{\mathrm{a}}$, J. Linnros ${ }^{\mathrm{a}}$ \\ ${ }^{a}$ Materials and Nanophysics, School of ICT, KTH Royal Institute of Technology, SE-164 40 Kista, \\ Sweden
}

\begin{abstract}
Direct measurements of the optical absorption cross section $(\sigma)$ and exciton lifetime are performed on a single silicon quantum dot fabricated by electron beam lithography (EBL), reactive ion etching (RIE) and oxidation. For this aim, single photon counting using, an avalanche photodiode detector (APD) is applied to record photoluminescence (PL) intensity traces under pulsed excitation. The PL decay is found to be of a mono-exponential character with a lifetime of $6.5 \mu \mathrm{s}$. By recording the photoluminescence rise time at different photon fluxes the absorption cross could be extracted yielding a value of $1.46 \times 10^{-14} \mathrm{~cm}^{2}$ under $405 \mathrm{~nm}$ excitation wavelength. The PL quantum efficiency is found to be about $9 \%$ for the specified single silicon quantum dot.
\end{abstract}

Keywords: Single silicon quantum dot, absorption cross section, quantum efficiency (QE), photoluminescence (PL) decay, luminescence rise time, silicon nanocrystals

\section{INTRODUCTION}

Quantum efficiency estimation of individual silicon quantum dots is of considerable importance, since it is one of the essential parameters influencing their applicability in optoelectronic and photonic devices. In order to determine the efficiency of an optical process in a nanocrystal, the physics of the photon absorption process as well as the emission mechanism must be well understood.

For years bulk silicon was considered as an inefficient light emitter due to its indirect band gap nature. After the discovery of strong photoluminescence (PL) emission of porous silicon at room temperature ${ }^{1,2}$, silicon nanocrystals became the center of interest for many researchers ${ }^{3,4}$. The quantum confinement model is believed to be relevant for silicon nanocrystals ${ }^{5,6}$, coupling the observed increase of the band gap energy with the decrease of nanocrystal size. The development of the single-dot spectroscopy technique ${ }^{7}$ led to a deeper insight of the photophysics of nanocrystals, however, many aspects of the light emission mechanisms are still not well understood. In particular, studies of photoluminescence absorption and decay have been mainly focusing on ensembles of silicon nanocrystals so far ${ }^{8,9,10}$, in which the effects of size and $\mathrm{Si}-\mathrm{SiO}_{2}$ interface nature of individual dots are averaged.

In the current work the results of PL lifetime and absorption cross section measurements of a single silicon quantum dot are presented. The samples were fabricated by electron beam lithography (EBL) and reactive ion etching (RIE), followed by self-limiting oxidation ${ }^{11}$. The well-defined positions of individual silicon quantum dots formed by this method enable repeatable single-dot spectroscopy. The measurements were carried out under different excitation laser power densities, using an avalanche photodiode detector (APD) for recording luminescence traces. Blinking and spectroscopy experiments were also performed on the same dot as complementary measurements. An estimate of the quantum efficiency of this nanocrystal, taking into account the detectivity of the PL setup, is presented.

\section{EXPERIMENTAL}

The experimental PL setup consists of a conventional wide field optical microscope system connected to a spectrograph (Andor, Shamrock 500i) and an EMCCD camera (Andor, iXon3) at one port and to an avalanche photodiode (Becker \& Hickl, DPC-230) at another port. A flipping mirror inside the microscope enables switching between these two ports.

*fatsan@kth.se; phone 468790 4159; fax 468 79043000;

Nanotechnology VI, edited by Rainer Adelung, Proc. of SPIE Vol. 8766, 876607

(C) 2013 SPIE · CCC code: 0277-786X/13/\$18 · doi: 10.1117/12.2017483 
Figure 1 shows a simple schematic of the PL setup. The sample is excited using a UV laser ( $405 \mathrm{~nm})$. Depending on the type of the PL measurements, the laser is operated in continuous or pulsed mode. For PL decay and absorption cross section measurements, pulsed excitation with a repetition rate of $20 \mathrm{kHz}$ and on-time duration of $10 \mu \mathrm{s}$ is used. This is done at different excitation laser power densities $\left(8-80 \mathrm{Wcm}^{-2}\right)$. On the other hand, for blinking measurements the PL intensity traces are recorded by the APD under continuous excitation $\left(8 \mathrm{Wcm}^{-2}\right)$. The laser beam has an angle of $\sim 30^{\circ}$ with respect to the sample surface and the emission is collected through a high numerical aperture lens $(\mathrm{NA}=0.7)$ with 100x magnification. Appropriate optical components such as high quality filters and mirrors are used to block background luminescence.

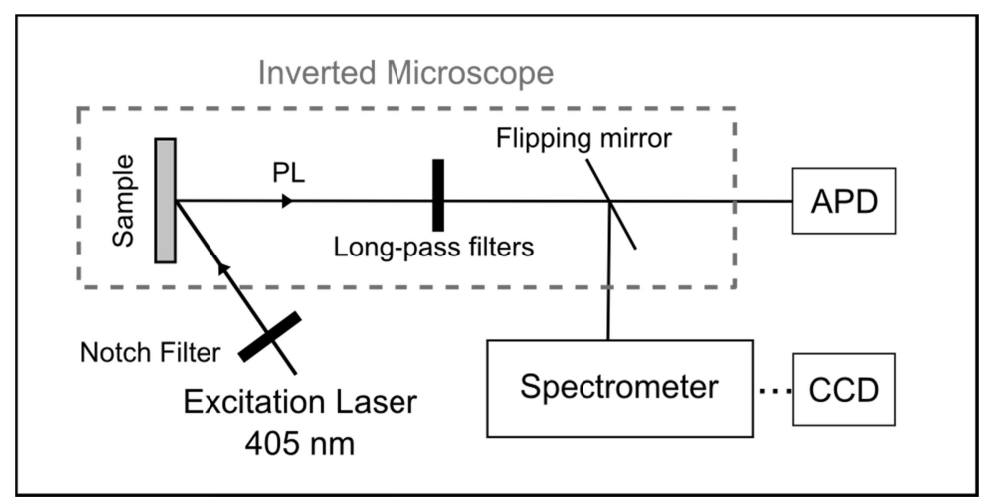

Figure 1. A Schematic of the experimental PL setup. The excitation laser beam ( $405 \mathrm{~nm})$ impinges on the sample surface at an angle of $\sim 30^{\circ}$. The emitted light is guided through an optical microscope and collected by an APD or spectrometer connected to an EMCCD camera for photon counting or spectral measurements, respectively.

The samples are fabricated in three steps. Electron beam lithography is used to define a pattern of undulating nano-walls on an n-type silicon wafer. Walls with three different thicknesses (40,65 and $90 \mathrm{~nm})$ are then formed by reactive ion etching (RIE). A simple schematic of one undulating nano-wall is shown in figure 2a. Finally, self-limiting oxidation inhomogeneously reduces the silicon structures, yielding individual silicon nanocrystals within the oxidized walls ${ }^{11}$. Due to large pre-selected separation between the dots $(\sim 2 \mu \mathrm{m})$, it is possible to perform several repeatable photoluminescence measurements on exactly the same dot. Applying short or long oxidation duration, single or double quantum dots can be formed in the thinnest and medium parts of the walls respectively ${ }^{11}$. Figure $2 \mathrm{c}$ shows a typical PL image of the sample after short oxidation and under cw UV excitation. Figure $2 b$ is an enlarged scanning electron microscope (SEM) image of the area specified by the gray rectangle in figure $2 \mathrm{c}$. All the measurements are focused on one single silicon quantum dot indicated by the red arrow in figure 2c.

\section{RESULTS}

Figure $2 \mathrm{~d}$ shows the room temperature PL spectrum of the single silicon quantum dot, specified by the red arrow in figure $2 \mathrm{c}$, under $8 \mathrm{Wcm}^{-2} \mathrm{cw}$ excitation power density. The emission energy peaks at $1.695 \mathrm{eV}$ with a line width of 100 $\mathrm{meV}$. This is typical for single silicon nanocrystals at room temperature ${ }^{6}$. The low-energy satellite peak at a distance of $60 \mathrm{meV}$ to the main peak is ascribed to TO phonon interactions. Previously it was shown that at lower temperatures the line-width of the main peak is narrower $(\sim 0.4 \mathrm{meV}$ at $10 \mathrm{~K})$ and the TO-phonon replica can be better resolved ${ }^{12,13}$. Blinking studies are also conducted on the same quantum dot based on single photon counting. For this purpose the PL intensity traces are recorded using an APD detector under continuous excitation of $8 \mathrm{Wcm}^{-2}$. For the particular dot studied, the emission intensity is relatively constant and blinking is only observed once at the end of the recorded trace, see Figure 3a. 


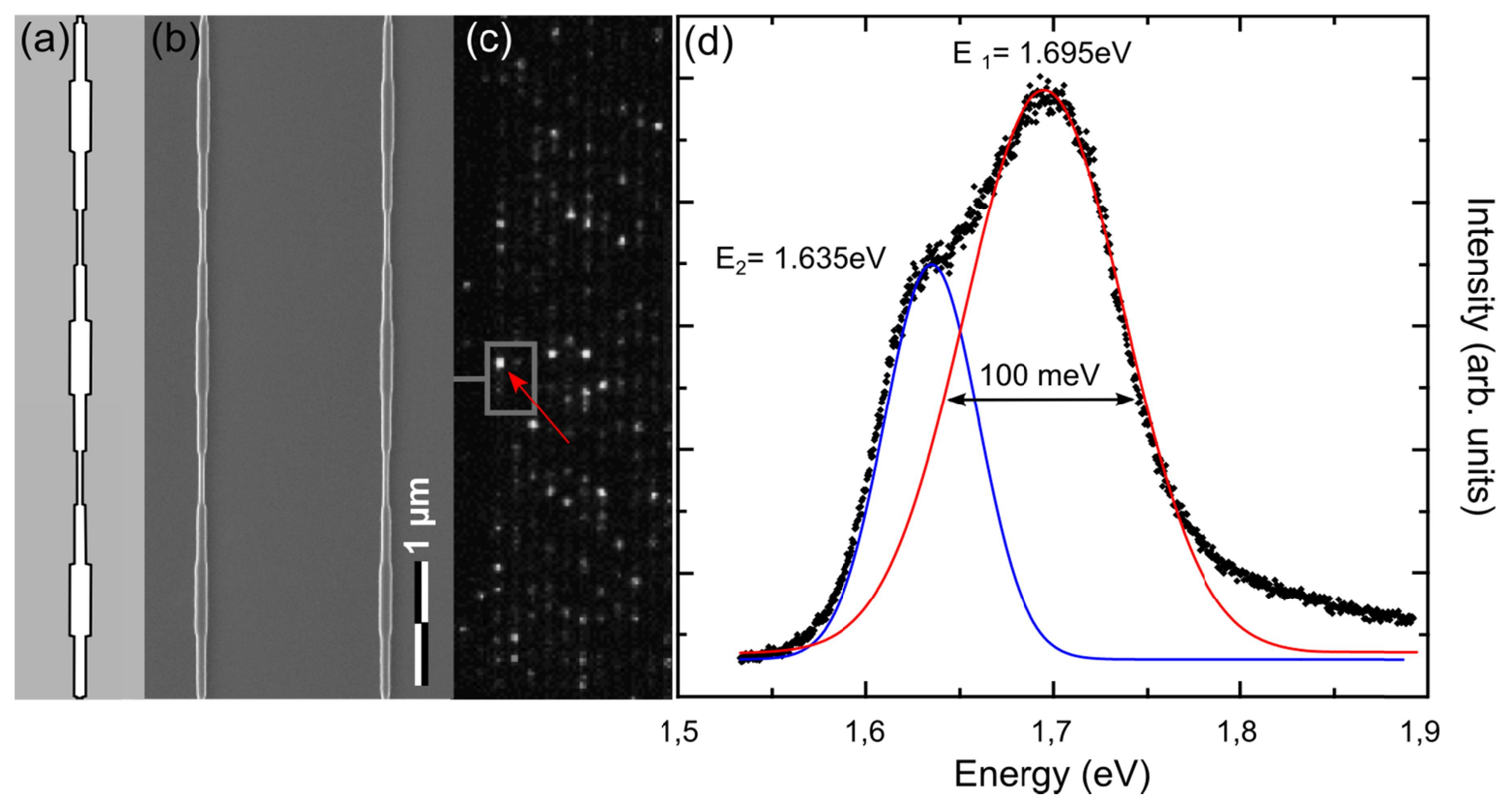

Figure 2. (a) Schematic of an undulating silicon nano-wall with three different thicknesses. (b) SEM image of such nanowalls after RIE and before oxidation. (c) Photoluminescence image of single silicon quantum dots formed inside the thinnest part of the walls after short oxidation ${ }^{11}$. The SEM image in (b) shows the details of the similar area determined by the grey rectangle. The single quantum dot under investigation is specified by a red arrow. (d) PL emission spectrum at room temperature and Gaussian fits to main (red) and satellite (blue) peak. Emission peaks at $1.695 \mathrm{eV}$ with full width half maximum (FWHM) of $100 \mathrm{meV}$ at an excitation power density of $8 \mathrm{Wcm}^{-2}$, the TO-phonon sideband is shifted $60 \mathrm{meV}$ to lower energy with respect to the main peak.

From this, two distinct intensity levels, denoted as ON and OFF, can clearly be defined from its histogram (Figure 3b). The dashed line in between ON and OFF indicates the threshold that is used for the distinction between the two states. Blinking statistics of similar single silicon quantum dots have been studied in detail in earlier work ${ }^{14}$. Blinking and spectral measurements confirm that the luminescent object under investigation is indeed a "single" silicon quantum dot.

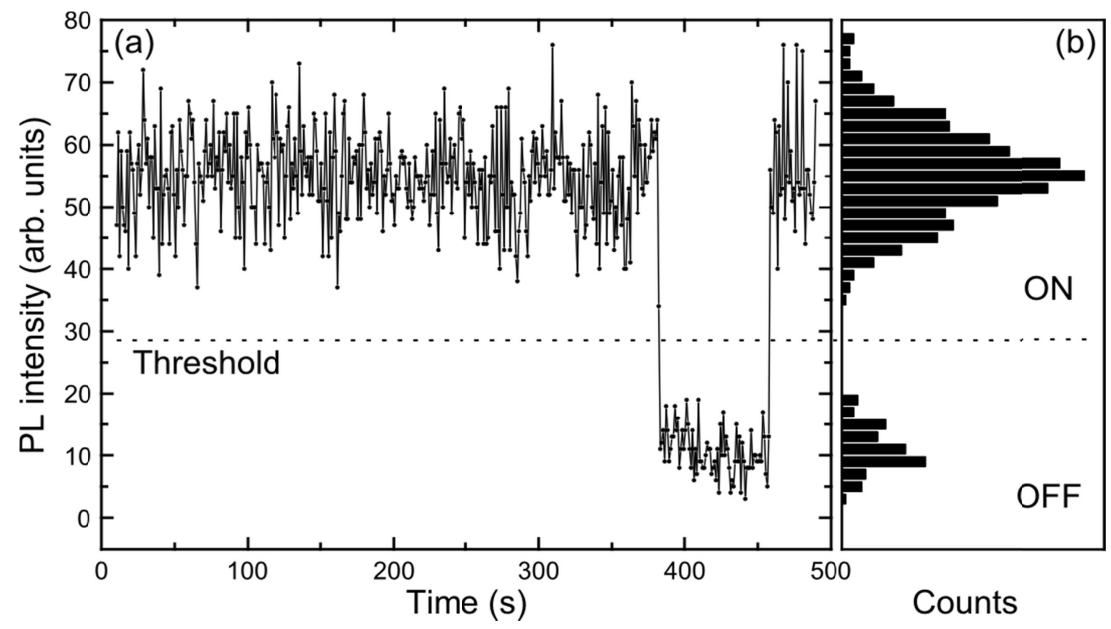

Figure 3. (a) PL Intensity traces of the studied single silicon quantum dot recorded at room temperature. (b) Corresponding histogram of the blinking traces, demonstrating the two distinct states ( $\mathrm{ON}$ and $\mathrm{OFF}$ ). A threshold line is considered in between the two levels. 


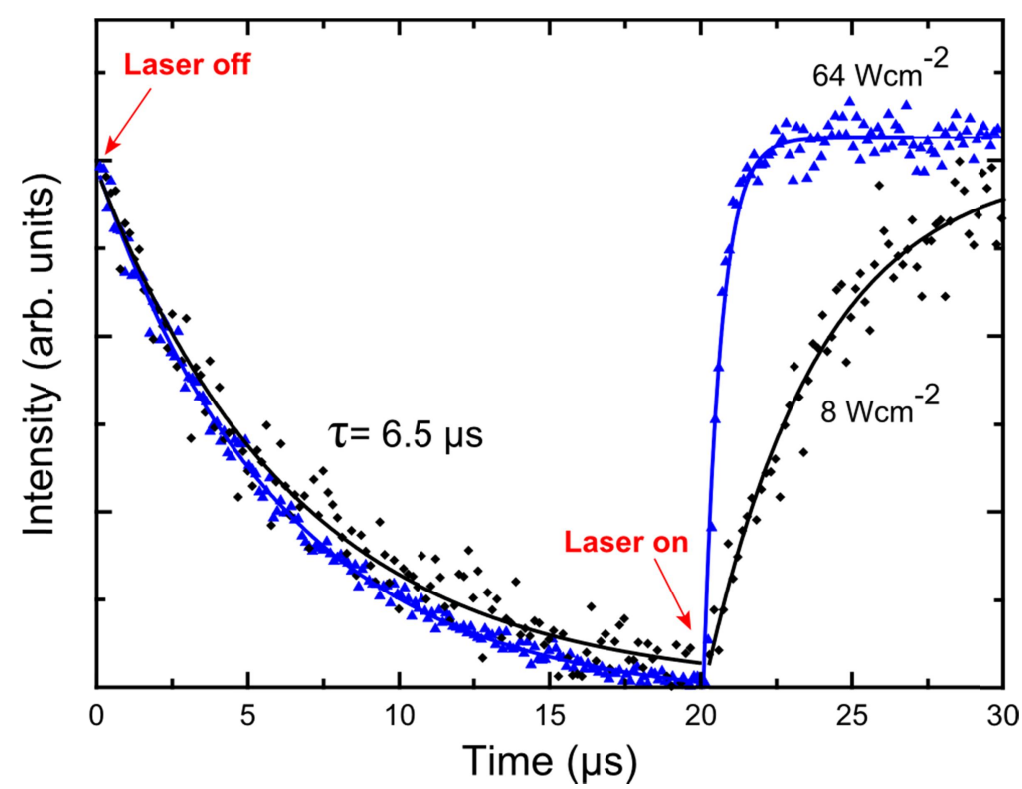

Figure 4. Photoluminescence decay and rise of the single silicon dot specified by the red arrow in figure $2 \mathrm{c}$, under two excitation power densities of 8 and $64 \mathrm{Wcm}^{-2}$. The solid lines are mono-exponential fits. (a) The photoluminescence decay exhibits a lifetime of $6.5 \mu \mathrm{s}$ for both laser power densities. whereas the rise time strongly depends on excitation.

The photoluminescence transient decay and rise is studied under pulsed laser excitation at a frequency of $20 \mathrm{kHz}$ using an on-time duration of $10 \mu \mathrm{s}$. The emitted photons are collected by the APD detector based on the single photon counting technique. The results are shown in figure 4 for two excitation power densities $\left(8\right.$ and $\left.64 \mathrm{Wcm}^{-2}\right)$.

A clear mono-exponential character with a lifetime of $\sim 6.5 \mu$ s can be observed for the PL decay under different excitation laser power densities. This is consistent with the results obtained from an earlier study on several single quantum dots performing time-resolved measurements ${ }^{15}$.

The kinetics of the luminescence rise, as the laser is switched on, follows a mono-exponential function, as well. In this case, the obtained rise time $\left(\tau_{\text {rise }}\right)$ values under 8 and $64 \mathrm{Wcm}^{-2}$ excitation power densities are $3.76 \mu \mathrm{s}$ and $0.49 \mu \mathrm{s}$, respectively.

Figure 5 shows the dependence of the luminescence rise rate on the excitation power density. A linear increase of the luminescence rise rate with excitation power can clearly be seen. This is expected from the equation of the temporal shape of the luminescence rise time ${ }^{16}$ :

$$
I(t)=I_{0}\left\{1-\exp \left[-\left(\sigma \phi+\frac{1}{\tau}\right) t\right]\right\}
$$

Where $\phi$ is the excitation flux and $\tau$ is the photoluminescence decay time.

Thus, the slope of the linear function in figure 5 yields the absorption cross section to a value of $\sigma=1.46 \times 10^{-14} \mathrm{~cm}^{2}$ for the specified single silicon quantum dot under $405 \mathrm{~nm}$ excitation. This is within an order of magnitude in agreement with the values reported by other groups ${ }^{9,10}$ from measurements on ensemble of silicon nanocrystals.

The luminescence quantum efficiency $(\mathrm{QE})$ is calculated using the formula for the excitation regime below saturation:

$$
I=P \times \sigma \times D \times Q E
$$

Where $\mathrm{P}$ is the excitation flux, $\sigma$ the absorption cross section and $\mathrm{D}$ the system detectivity ${ }^{17}$.

The system detectivity is measured for different optical components in the PL setup as the percentage of efficient light transmission/reflection through each of them. Approximately $10 \%$ of the light is considered to be emitted efficiently 
into the upper hemisphere of the single quantum dot from the surface ${ }^{18}$. Finally the obtained quantum efficiency is $\sim 9 \%$, which is close to a previously reported value $(\sim 15 \%)^{19}$.

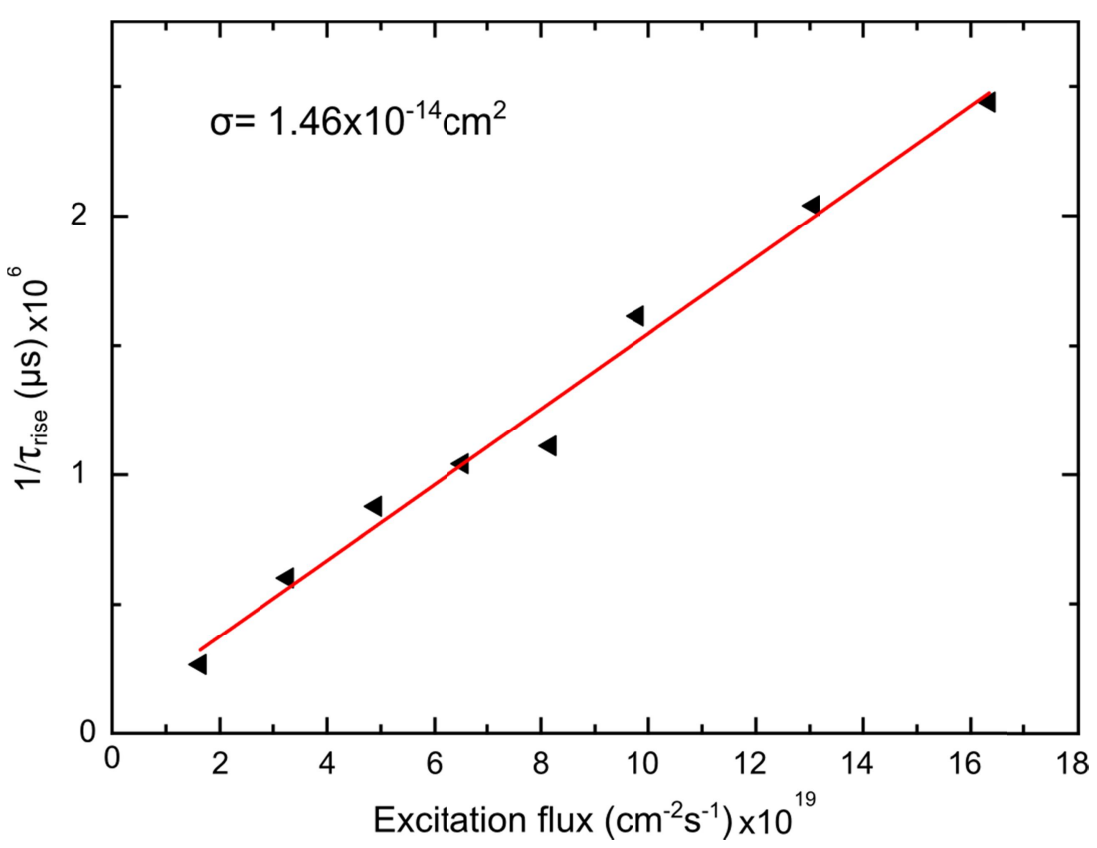

Figure 5. Reciprocal of the luminescence rise time versus photon flux under the saturation level. The luminescence rise rate is a linear function of the excitation flux.

\section{CONCLUSION}

Measurements of luminescence decay and rise time under different laser excitation power densities on an individual silicon quantum dot, using the photon counting technique, were performed. Two distinct emission intensity states, as well as spectral measurements, confirm the observation of a single silicon quantum dot. The decay time is of monoexponential character with $6.5 \mu$ s decay time, which is in agreement with results obtained from time-resolved PL decay measurements on similar single silicon quantum dots ${ }^{15}$. The slope of the linear increase of the luminescence rise rate yields an absorption cross section value of $1.46 \times 10^{-14} \mathrm{~cm}^{2}$. The measured value of the absorption cross-section allowed us to estimate the quantum efficiency of this particular dot to be $\sim 9 \%$.

It could be shown that luminescence transients can be successfully measured for a single silicon nanocrystal. This enables studies on many individual objects and subsequent comparison of their properties amongst each other, as well as to ensembles. Individual variations between single emitters can be resolved and possibly correlated to their structural differences in future work. In addition, the density of states of a nanocrystal can possibly be probed using this technique by applying different excitation wavelengths. 


\section{REFERENCES}

[1] Canham, L. T., "Silicon quantum wire array fabrication by electrochemical and chemical dissolution of wafers,"Appl. Phys. Lett. 57, 1046-1048 (1990).

[2] Lehmann, V. and Gösele, U., "Porous silicon formation: A quantum wire effect," Appl. Phys. Lett. 57, 856-858 (1991).

[3] Kovalev, D., Heckler, H., Polisski, G. Diener, J. and Kock, F., "Optical properties of silicon nanocrystals," Optical Materials 17, 35-40 (2001).

[4] Meier, C., Gondorf, A., Lüttjohann, S., Lorke, A. and Wiggers, H., "Silicon nanoparticles: Absorption, emission, and the nature of the electronic bandgap," J. Appl. Phys. 101, 103112 (2007).

[5] Wilson, W. L., Szajowski, P. F. and Brus, L. E., "Quantum Confinement in Size-Selected, Surface-Oxidized Silicon Nanocrystals," Science 19, 1242-1244 (1993).

[6] Valenta, J., Juhasz, R. and Linnros, J., "Photoluminescence spectroscopy of single silicon quantum dots," Appl. Phys. Lett. 80, 1070-1072 (2002).

[7] Moerner, W. E. and Fromm, D. P., "Methods of single-molecule fluorescence spectroscopy and microscopy," Rev. Sci. Instrum. 74, 3597-6319 (2003).

[8] Linnros, J., Lalic, N., Galeckas, A. and Grivickas, V., "Analysis of the stretched exponential photoluminescence decay from nanometer-sized silicon crystals in $\mathrm{SiO}_{2}$," J. Appl. Phys. 86, 6128-6134 (1999).

[9] Kovalev, D., Diener, J., Heckle, H., Polisski, G., Kunzner, N. and Kock, F., "Optical absorption cross sections of silicon nanocrystals, "Phys. Rev. B 61, 4485-4487 (2000).

[10] Garcia, C., Garrido, B., Pellegrino, P., Ferre, R., Moreno, J. A., Morante, J. R., Pavesi, L. and Cazzanelli, M., "Size dependence of lifetime and absorption cross section of Si nanocrystals embedded in $\mathrm{SiO}_{2}$," Appl. Phys. Lett. 82, 1595-1597 (2003).

[11]Bruhn, B., Sangghaleh, F. and Linnros, J., "Fabricating single silicon quantum rods for repeatable single dot photoluminescence measurements," Phys. Status Solidi A 208, 631-634 (2011).

[12] Sychugov, I., Valenta, J., Mitsuishi, K., Fujii, M. and Linnros, J., "Photoluminescence measurements of zerophonon optical trasitions in silicon nanocrystals," Phys. Rev. B 84, 125326 (2011).

[13] Kovalev, D., Heckler, H., Ben-Chorin, M., Polisski, G., Schwartzkopff, M. and Koch, F., "Breakdown of the kconservation rule in Si nanocrystals," Phys. Rev. Lett. 81, 2803-2806 (1998).

[14] Bruhn, B., Valenta, J., Sangghaleh, F. and Linnros, J., "Blinking statistics of silicon quantum dots," Nano lett. 11, 5574-5580 (2011).

[15] Sangghaleh, F., Bruhn, B., Schmidt, T. and Linnros, J., "Exciton lifetime measurements of single silicon nanocrystals," Nanotechnology, in press (2013).

[16] Priolo, F., Franzo, G., Pacifici, D., Vinciguerra, V., Iacona, F. and Irrera, A. "Role of the energy transfer in the optical properties of undoped and Er-doped interacting Si nanocrystals," J. Appl. Phys. 89, 264-272 (2001).

[17] Valenta, J., Juhasz, R. and Linnros, J., "Photoluminescence from single silicon quantum dots at room temperature," Journal of Luminescence 98, 15-22 (2002).

[18] Sychugov, I., Omi, H. and Kobayashi, Y., "On the role of the substrate in light-harvesting experiments," Optics Lett. 33, 1807-1809 (2008).

[19] Sychugov, I., Galeckas, A., Elfström, N., Wilkinson, A. R., Elliman, R.G. and Linnros, J., "Effect of substrate proximity on luminescence yield from Si nanocrystals," Appl. Phys. Lett. 89, 111124 (2006).

[20] Garrido Fernandez, B., López, M., García, C., Pérez-Rodríguez, A., Morante, J. R., Bonafos, C., Carrada, M. and Claverie, A., "Influence of average size and interface passivation on the spectral emission of Si nanocrystals embedded in $\mathrm{SiO}_{2}$ '" J. Appl. Phys. 91, 798-807 (2002). 